\title{
Sampling Based Vehicle Motion Planning for Autonomous Valet Parking with Moving Obstacles
}

\author{
Yonghwan Jeong ${ }^{1)}$ Seonwook Kim ${ }^{1)}$ Byeong Rim Jo ${ }^{2)}$ Hyunseok Shin ${ }^{2)}$ Kyongsu Yi ${ }^{1)}$ \\ 1) Seoul National University, School of Mechanical and Aerospace Engineering \\ 301-dong 113-ho Gwanak-ro, Gwanak-gu, Seoul, 08826, Republic of Korea (E-mail: winqq1234@snu.ac.kr) \\ 2) LG Electronics, Vehicle Component Technology, Autonomous Vehicle Technology Lab \\ 19, Yangjae-daero 11-gil, Seocho-gu, Seoul, 06772, Republic of Korea
}

Received on October 12, 2017

\begin{abstract}
This paper describes a motion planning algorithm for unstructured dynamic environments with motion prediction for moving obstacles. The proposed algorithm is composed of the four steps: 1) target motion prediction; 2) drivable area decision 3) local path planning and 4) vehicle control. The target motion prediction is crucial parts for realizing autonomous valet parking system because many vehicles which search available parking lot exist simultaneously. To predict future motion of target, the intention of the target should be inferred first. Interacting multiple model (IMM) filter using two models has been used to infer the intention of the target. Based on the inferred intention, most appropriate model's results are used as a predicted trajectory of the target vehicle. After that, the drivable area is decided to avoid collision with static obstacles and moving targets using potential filed approach to assessment the risk. In this stage, pre-defined parking lot map which contains boundary of the parking lots and waypoints is used to define initial guess of the drivable area. Inside the drivable area, rapidly-exploring random tree (RRT) generate the desired local path while guaranteeing the real-time performance in dynamic environments. Finally, path tracking controller and speed controller calculate desired steering wheel and longitudinal acceleration input. The proposed motion planning algorithm is validated via MATLAB based computer simulation. Simulation results demonstrate the ability of the proposed motion planning algorithm for unconstructed dynamic environments to plan collision-free path which is appropriate in parking lot situations.
\end{abstract}

KEY WORDS: Electronics and Control, Target Intention Inference, Integrating multiple model, Potential field, Rapidlyexploring random tree, Motion planning, Motion prediction, Autonomous valet parking [E1]

\section{Introduction}

In recent years, there has been an increasing amount of research on the subject of advanced driver assistance systems (ADAS) and autonomous vehicle. Among these technologies, ADAS have been an important research and development topic because ADAS is close to mass production. Especially, park assistant system is one of the most close to mass production technology since parking task is troublesome task which takes lots of time. Therefore, a lot of park assistant systems has been studied in industry and academia. A variety of sensors and infrastructure of the parking lot have been used to develop park assistant system ${ }^{(1-5)}$. Basically, passive safety system which detect obstacles while parking is the most commercialized parking assist system which is commercialized almost thirty years ago ${ }^{(6,7)}$. About 10 years ago, active park assist system such as smart parking assistant system (SPAS) has been produced to improve convenience of the driver ${ }^{(8)}$. However, these park assist system only give a steering assist when tracking the parking path and parking space for SPAS should be founded by driver. Therefore, autonomous valet parking system has been extensively researched to remedy former parking assistant system's shortcomings, improve the driver's convenience and enhance the safety in parking lot.

Numerous approaches to address the path planning problem have been proposed in the literature. The most representative types of the path planning algorithm are sampling-based, optimization based method, and potential field approach. For sampling-based method, rapidly-exploring random tree (RRT) algorithm were often used which guarantees the existence of the desired path ${ }^{(9,10)}$. These RRT method has been modified to increase their performance and feasibility by combining the probabilistic approach or closed-loop prediction. Recently, Model Predictive Control (MPC) has an attractive method for solving the path planning and control problem because of its capability to systematically handle system nonlinearities and constraints ${ }^{(11-15)}$. A potential field approach has been successfully implemented for robot motion planning and path tracking so far ${ }^{(16)}$, and the concept has been applied on an integrated safety system and automated vehicle navigation ${ }^{(17-19)}$. Yoon et al. presents the $A^{*}$ algorithm using two heuristics which consider the kinematics of a vehicle with and without obstacles respectively ${ }^{(20)}$. Compared to the previous researches that handle specific parking scenarios only, Bai Li and Zhijiang Shao proposed motion planning in the presence of irregularly placed obstacles. various kinds of parking cases in a unified way regardless they are regular parking scenarios (e.g., parallel, perpendicular or echelon parking cases) or not ${ }^{(21)}$.

In this research, we focus on designing a motion planning algorithm for autonomous valet parking system in dynamic environments based on the potential field and the rapidly- 
exploring random tree (RRT) approach. The proposed algorithm is composed of target inention inference, drivable area decision, and local path planning. The target intentin inference algorithm uses only position and heading angle of target vehicle to predict the future trajectory of the target. To do this, interacting multiple model (IMM) filter is used to find approapriate motion model of the target. After that potential field approach and parking lot map are used to determine drivable area which functions as boundary of local path generation. The local path planning algorithm based on RRT guarantees the real-time performance of the local path generation and collision avoidance in dynamic environments while considering the feasibility of the generated local path. In this step, concept of seeding surface and probability distribution function of sampling is adopted to improve the real-time performance which is hard to achieve when using normal RRT. After the desired local path is generated, a path tracking controller which determines a desired steering wheel angle is designed by the combination of road curvature based feedforward and feedback linear quadratic optimal control method using 2 DOF bicycle model. The developed motion planning algorithm is validated via MATLAB based computer simulation.

This paper is constituted as follows: the section 2 presents an overall architecture of the proposed motion planning algorithm for autonomous valet parking system. In section 3, local path planning algorithm for collision avoidance with static and moving obstacle is described. Then Section 4 shows the MATALB based simulation results to validate the proposed motion planning algorithm. Finally, the contribution of this research and the future works are in section 5 .

\section{Overall Architecture}

The overall architecture of the proposed motion planning algorithm, which consists of four steps, target motion prediction, drivable area decision, local path planning and vehicle control, is shown in Fig. 1. In the target motion prediction stage, which uses position and heading angle of the target vehicle, determines the intention and predicts the future trajectory of target vehicle. To do this, IMM filter has been adopted to infer the inention of the target and simple vehicle model with virtual goal is used to predict the future trajectory of the target. Second, the drivable area decision uses potential field approach to assess the risk of a static and moving obstacles with differenct potential repulsive energy functions. Next, local path planning uses rapidly-exploring random tree (RRT) algorithm to generate the local desired path while guaranteeing the real-time performance and feasibility of the generated local path. Closed-loop vehicle trajectory prediction has been used to find out whether generated local path is possible to track. Finally, to tracking the path, the controller is designed to determine the desired steering wheel angle and desired throttle and brake input. The steering input and the desired throttle/brake input is determined using LQR approach and adaptive controller respectively ${ }^{(22)}$.

\section{Local Path Planning}

\subsection{Target Motion Prediction}

To predict the future motion of the target, the intention of the target should be inferred first. Because the intention of the target vehicles is the most important factor which must be known to predict the future motion of the target. In this paper, the intention of the target vehicles means possible behaviors which can be taken by target such as going straight, turning left, turning right, or avoiding the oncoming vehicle. If intention inference of the vehicle in public road is the object of this research, discrimination between lane keeping, lane changing, and turning is a core function that should be prepared before developing motion planning algorithm. However, in parking lot, the concept of the lane is not exist which is the core structure of the road. Which means that distinguishing lane keeping and lane changing is less important than distinguishing going straight and avoiding the oncoming vehicle. Therefore, target intention inference algorithm is designed to determine whether going straight or avoiding ego vehicle.

In this paper, IMM filter has been used to infer the intention of target vehicle using relative position and heading angle which can be obtained from laser scanner. To design IMM filter, proper process model for local Kalman filter should be defined to obtain estimation performance of IMM filter. When the vehicle is

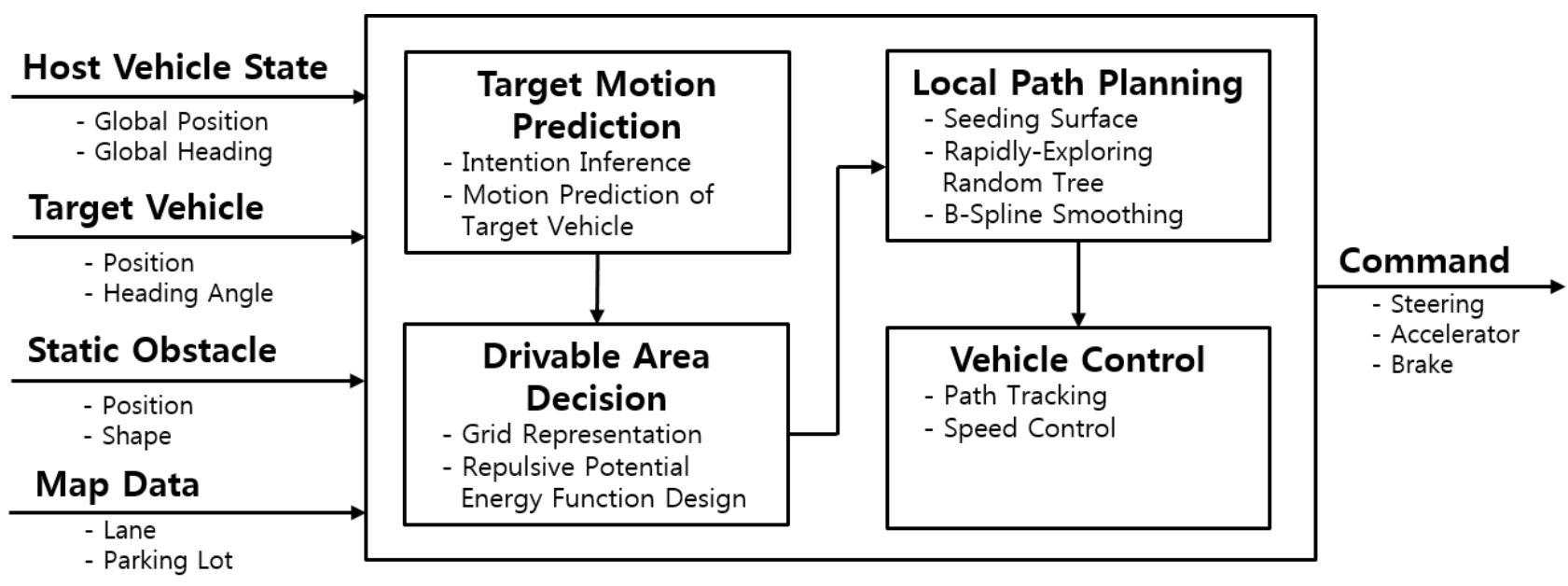

Fig. 1 Overall Architecture of Proposed Parking Algorithm. 
moving in the parking lot, it is unnecessary to express the behavior or the vehicle as a dynamic model because tire slip and body slip is negligible in low speed region. The motion of the target vehicle is modeled in local coordinate of ego vehicle as follows :

$$
\begin{aligned}
& v_{x}(t)=v(t) \cos \theta(t) \\
& v_{y}(t)=v(t) \sin \theta(t) \\
& a_{x}(t)=a(t) \cos \theta(t)-v(t) \cdot \gamma(t) \sin \theta(t) \\
& a_{y}(t)=a(t) \sin \theta(t)+v(t) \cdot \gamma(t) \cos \theta(t)
\end{aligned}
$$

where, $\theta, v, a$, and $\gamma$ represent the heading angle, velocity, acceleration, and yaw-rate of the target respectively. To define model, primary differential value of the $a$ and $\gamma$ should be determined to derive the derivative of above equations. In this research, derivative of the $a$ and $\gamma$ is assumed as exponential function with decay rate $k_{a}$ and $k_{r}$ to describe the natural reduction of $a$ and $\gamma$ and easily differentiate the equations. It is a reasonable assumption because drivers generally not maintain acceleration or yaw-rate a certain value but converge to zero finally. Therefore, the differential equation of acceleration and yaw-rate can be assumed as follows:

$$
\begin{aligned}
& \dot{a}(t)=a(t) \cdot \exp \left(-k_{a} \cdot t\right) \\
& \dot{\gamma}(t)=\gamma(t) \cdot \exp \left(-k_{\gamma} \cdot t\right)
\end{aligned}
$$

The decay rate $\left(k_{a}, k_{r}\right)$ are set as $(0,0)$ for going straight model and $(0.05,0.1)$ for avoiding the oncoming vehicle model each. By discretizing the above 6 equations, discrete model for local Kalman filter is derived as

$$
\begin{aligned}
p_{x, k+1}= & p_{x, k}+v_{k} \cos \theta_{k} \cdot d t \\
& +\left(a_{k} \cos \theta_{k}-v_{k} \cdot \gamma_{k} \sin \theta_{k}\right) \cdot d t^{2} / 2 \\
p_{y, k+1}= & p_{y, k}+v_{k} \sin \theta_{k} \cdot d t \\
& +\left(a_{k} \sin \theta_{k}+v_{k} \cdot \gamma_{k} \cos \theta_{k}\right) \cdot d t^{2} / 2 \\
\theta_{k+1}= & \theta_{k}+\gamma_{k} \cdot d t+\dot{\gamma}_{k} d t^{2} / 2 \\
v_{k+1}= & v_{k}+a_{k} \cdot d t+\left(-k_{a}\right) a_{k} \cdot d t^{2} / 2 \\
\gamma_{k+1}= & \gamma_{k}+\dot{\gamma}_{k} \cdot d t+\left(-k_{\gamma}\right) \dot{\gamma}_{k} \cdot d t^{2} / 2 \\
a_{k+1}= & a_{k}+\left(-k_{a}\right) a_{k} \cdot d t+\left(-k_{a}{ }^{2}\right) a_{k} \cdot d t^{2} / 2 \\
\dot{\gamma}_{k+1}= & \dot{\gamma}_{k}+\left(-k_{\gamma}\right) \dot{\gamma}_{k} \cdot d t+\left(-k_{\gamma}{ }^{2}\right) \dot{\gamma}_{k} \cdot d t^{2} / 2
\end{aligned}
$$

Among these difference equations, initial yaw-rate at $k$-th step $\gamma_{k}$ should be defined differently for each model. A virtual goal, which is described as red cross marker in Fig. 4, is assumed for each model to define $\gamma_{k}$ in each step of prediction horizon. For going straight model, virtual goal is simply defined as an end position of the straight line. However, for avoiding the oncoming vehicle models, virtual goal is located at the center of ego vehicle and road boundary with opposite heading angle of ego vehicle.
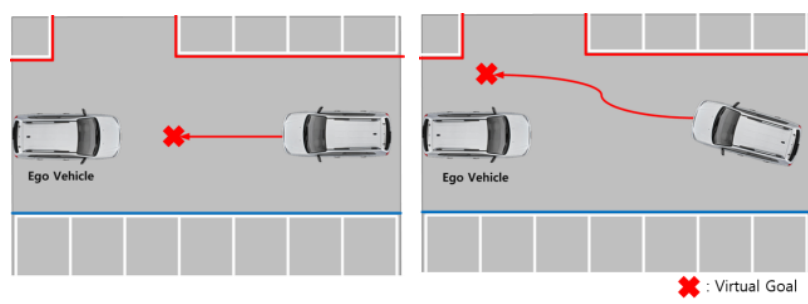

Fig. 2 Vehicle Motion Models for Target Intention Inference Algorithm.

After all virtual goals are defined, center of the target vehicle and virtual goal is connected using $3^{\text {rd }}$ order polynomial and calculate $\gamma_{k}$ as follows :

$$
\begin{aligned}
& \gamma_{k}=\left|\frac{\left(1+\dot{y}_{k}^{2}\right)^{3 / 2}}{\ddot{y}_{k}}\right| \\
& \text { where, } y_{k}=a_{1, k} x_{k}^{3}+a_{2, k} x_{k}^{2}+a_{3, k} x_{k}+a_{4, k}
\end{aligned}
$$

Using these models, IMM filter has been designed in a commonly used form ${ }^{(23)}$ and the model which has a highest probability is selected as an inferred intention of the target vehicle. After intention is estimated, same model is used to predict a trajectory of target vehicle in 0 to 2 second prediction horizon with $100 \mathrm{~ms}$ sampling time. The example of the target motion prediction is described in Fig. 3. The ego and oncoming target vehicle is represented as red and green vehicle respectively in Fig. 3. In this example, the oncoming target vehicle is moved left side of the ego vehicle and try to pass the ego vehicle. The probability of going straight model is highest and the future trajectory of oncoming target is predicted as black line started from the center of green vehicle. If the proposed IMM filter is tuned with unappropriate $\left(k_{a}, k_{r}\right)$, a detecting the transition between turning and going straight motion is difficult. Because diminishing of acceleration and yaw-rate are refected in IMM model by using the proposed exponential function based differential equation

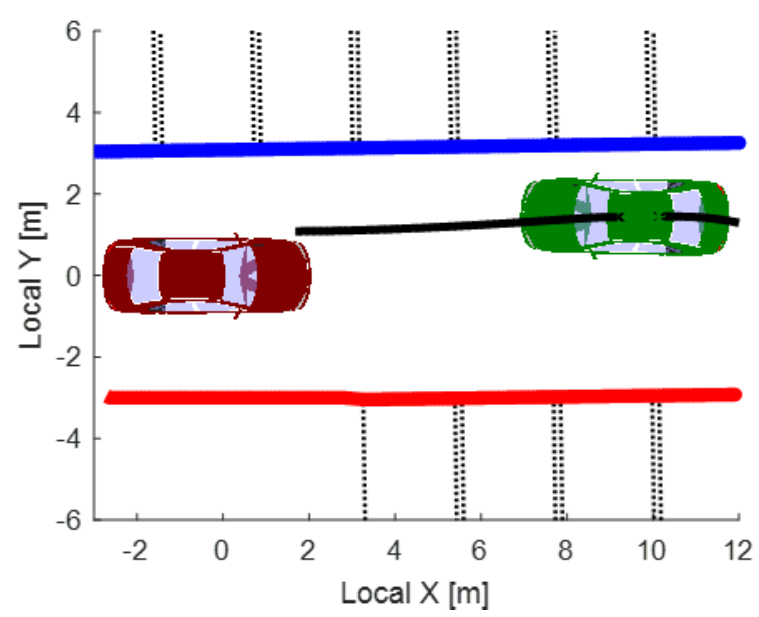

Fig. 3 The example of target motion prediction 


\subsection{Drivable Area Deicision}

To generate a desired local path considering unconstructed environments, drivable area decision with risk assessment should be conducted first. Potential filed approach has been adopted to estimate the collision risk between a predicted trajectory of target vehicle, road boundary with static obstacles and a desired local path of ego vehicle. Which means that predicted trajectory of target vehicle and road boundary with static obstacles are used as inputs of the repulsive potential energy function. In this process, predicted trajectory is obtained from target intention inference algorithm which is mentioned in chapter 3.1 and road boundary is stored in parking lot map which is pre-defined before like Fig. 4. When we evaluating repulsive potential energy based on information from environment sensors and parking lot map, different function is required for static and moving obstacle respectively to deal with the uncertainty of the moving obstacles. In this paper, only Euclidian distance is considered for static obstacle case and relative position and velocity are used for moving obstacle case. Also, to calculate the potential function, area around the ego vehicle is represented by a grid form to reduce the computation load as shown in Fig. 5. First, repulsive potential energy function for static obstacles is designed as follow :

$$
\text { if } \begin{aligned}
& D<Q \\
& U_{\text {static }}=\min \left[U_{\max }, k_{1} \cdot\left(\frac{1}{D}-\frac{1}{Q}\right)^{2}\right]
\end{aligned}
$$

else

$$
U_{\text {static }}=0
$$

where, $U_{\max }$ and $k_{1}$ denotes maximum value of repulsive potential energy and tuning gain respectively. $Q$ and $D$ denotes region of interest for repulsive potential energy calculation and Euclidian distance from static obstacle to grid point each. This equation means that collision risk with static obstacle increase in proportion to the inverse of the Euclidian distance and if ego vehicle have a safety distance above a certain level, $Q$, collision risk can be assumed as a zero value.

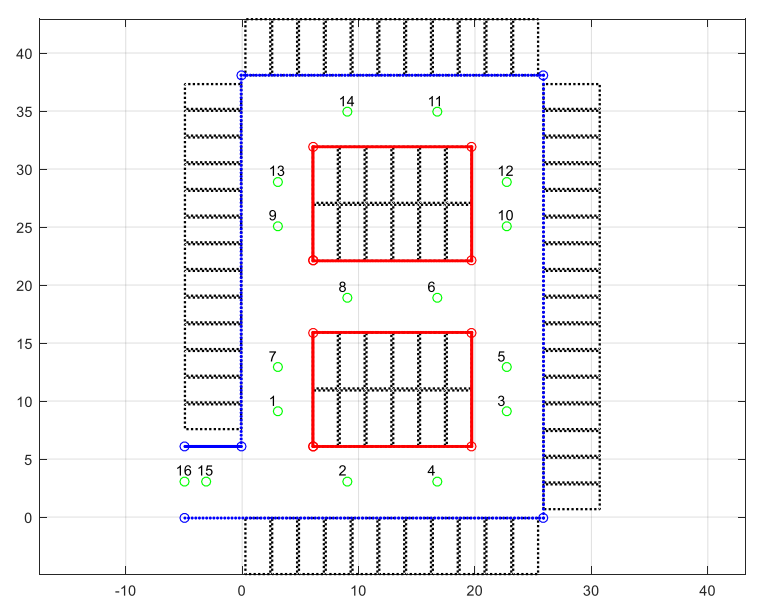

Fig. 4 Parking Lot Map.

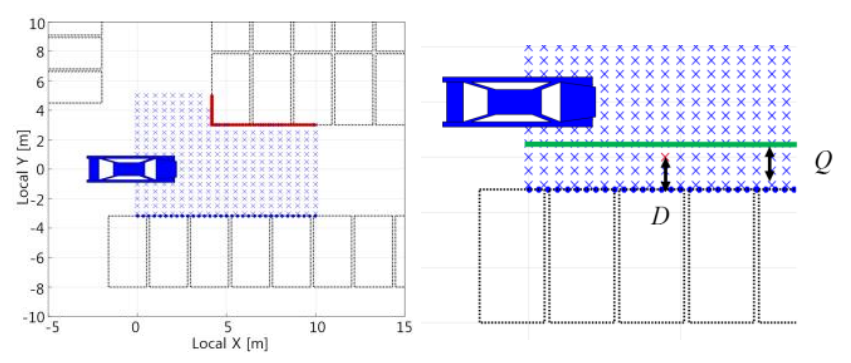

Fig. 5 Grid Representation for Calculation of Repulsive Potential Energy Function

Next, repulsive potential energy function for moving obstacles is designed as follow :

$$
\begin{aligned}
& \text { if }\|\rho\|<\rho_{R O I} \\
& U_{\text {mov }}=\min \left[U_{\max }, k_{2} \cdot \exp \left(-\left(\frac{x_{r, p}}{\dot{x}_{r, p}}\right)^{2}-\left(\frac{y_{r, p}}{\dot{y}_{r, p}}\right)^{2}\right)\right]
\end{aligned}
$$

else

$$
U_{\text {mov }}=0
$$

where, $x_{r, p}$ and $y_{r, p}$ denotes relative $\mathrm{x}$ and $\mathrm{y}$ axis position in local coordinate of ego vehicle respectively. $\dot{x}_{r, p}$ and $\dot{y}_{r, p}$ is derivative of the $x_{r, p}$ and $y_{r, p}$, which means that these are relative velocity of the target vehicle in local coordinate. Also $\rho$ is the shortest distance to moving obstacle's predicted trajectory and $\rho_{R O I}$ is the region of interest from predicted trajectory of moving target. The sum of $U_{\text {static }}$ and $U_{m o v}$ is used as a repulsive potential energy function for local path generation.

The results of the drivable area decision is described in Fig. 6. As shown in Fig. 6, area inside the region of interest is discretized as $50 \mathrm{~cm}$ size grid to reduce computation load when calculating repulsive potential energy function. High risk region is marked as yellow grid and safe region is marked as blue grid. Also drivable area is defined as boundary of maximum potential value which is marked as a black dotted line.

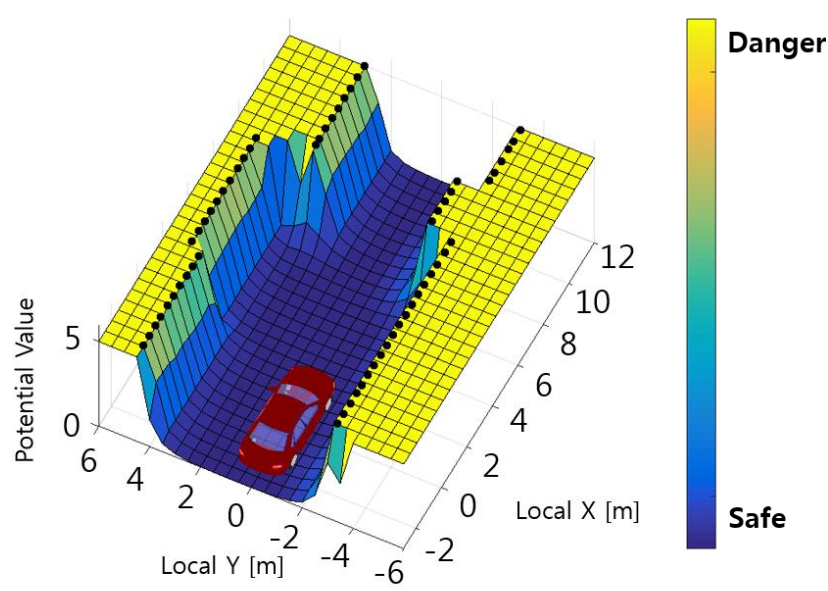

Fig. 6 Drivable Area Representation and Risk Assessment Using Potential Field Appproach 


\subsection{RRT based Local Path Generation}

Potential field approach generally create desired path along the local minimum of the potential energy. However, these method could be generate unfeasible path and increase a computation load because all area inside region of interest are considered. Therefore, key considerations of the local path generation are reflecting the limitation of the vehicle dynamics and reducing computation load to achieve real-time performance. To satisfy these considerations, area inside the region of interest is divided in several surfaces based on the global path which is assumed that global path is given form the parking lot map and infrastructure. The desired local path is defined using modified random rapidly-exploring random tree (RRT), which connects random samples on the these surfaces. In this paper, these surfaces are called seeding surface because random samples are generated on them just like planting in the furrow. This concept is described in Fig. 7. Based on this concept, potential value results using chapter 3.2 equations is described as 3-dimentional surface and representative three surfaces are shown in Fig. 8. Global path is shown as cyan solid line and seeding surfaces are represented as red solid curves.

After dividing the area in several seeding surfaces, random sampling should be taken on that surfaces. To reduce the number of samples, collision risk is converted as a probability density function of the random samples. As mentioned before, collision risk is evaluated as a potential value which is calculated in chapter 4.2 , which means that possibility of desired local path generation to lower potential value region is higher than other cases. To do this, the inverse of the potential value is used as probability density function. Before using the inverse of the potential value as a probability density function, these inverses should be normalized to satisfy the definition of probability density function. If we define $y$ as a random variable using the inverse of potential value as potential density function, mapping random variable $y$ to normal random variable $x$ is needed. Using the normalization condition of random variable, we can obtain random number $y$ using uniform distribution $x$. This process can be summarized as follows :

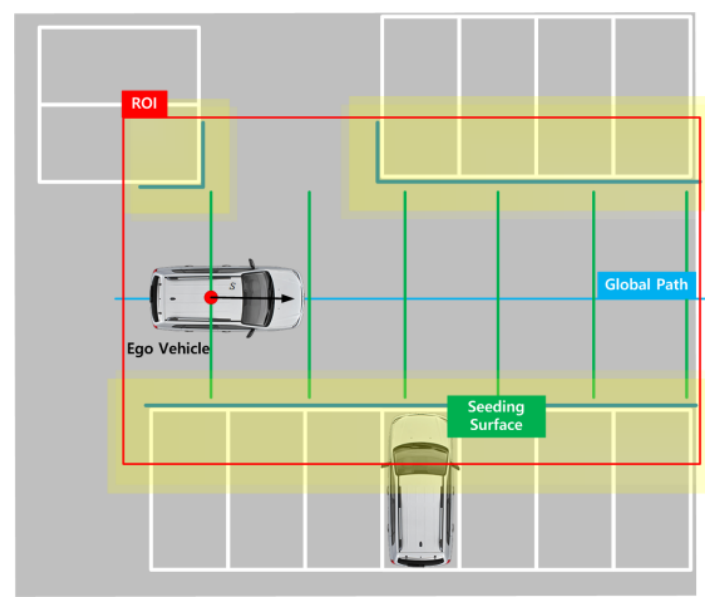

Fig.7 Seeding Surface Configuration

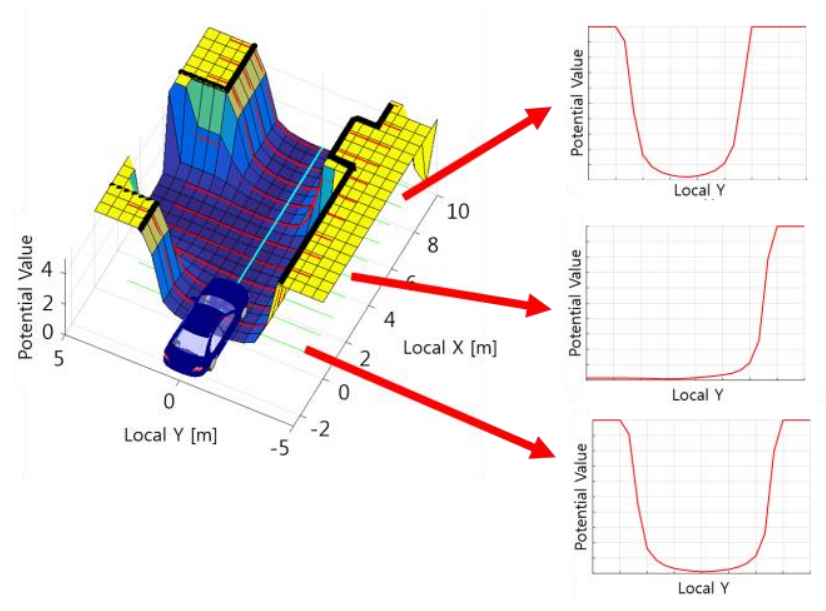

Fig. 8 Cross Section of the Potential Field

$$
\begin{aligned}
& y=f(x) \\
& \begin{aligned}
\int_{0}^{y} \frac{d P}{d y} d y & =\int_{0}^{x(y)} \frac{d P}{d x} d x \\
& =x(y)
\end{aligned}
\end{aligned}
$$

where, $P$ denotes cumulative distribution function. This probability density function is used to generate candidate samples for modified RRT algorithm to reduce the sample numbers than conventional RRT which grows in any direction randomly. After samples are generated, random tree grows using a cost which is defined as

$$
C_{k, k+1}(i, j)=k_{1} \cdot d_{k, k+1}(i, j)+k_{2} \cdot l_{k+1}(j)+k_{3} \cdot c_{k+1}(j)
$$

The example of the cost function calculation is described in Fig. 9. $L_{k}$ is assigned to $k$-th seeding surfaces which has a $n$ samples and index $i, j$ is denoted as $i$-th sample of $k$-th seeding surface and $j$-th sample of $k+1$-th seeding surface respectively. $d_{k, k+1}(i, j)$ means travel distance between two samples on $k$ and $k+1$-th seeding surface and $l_{k+1}(j)$ is the clearance to drivable area boundary from $j$-th sample of $k+1$-th seeding surface. $c_{k+1}(j)$ is the deviation from global path. The balance between travel distance and clearance is adjusted by $k_{1}, k_{2}$ and $k_{3}$. Using

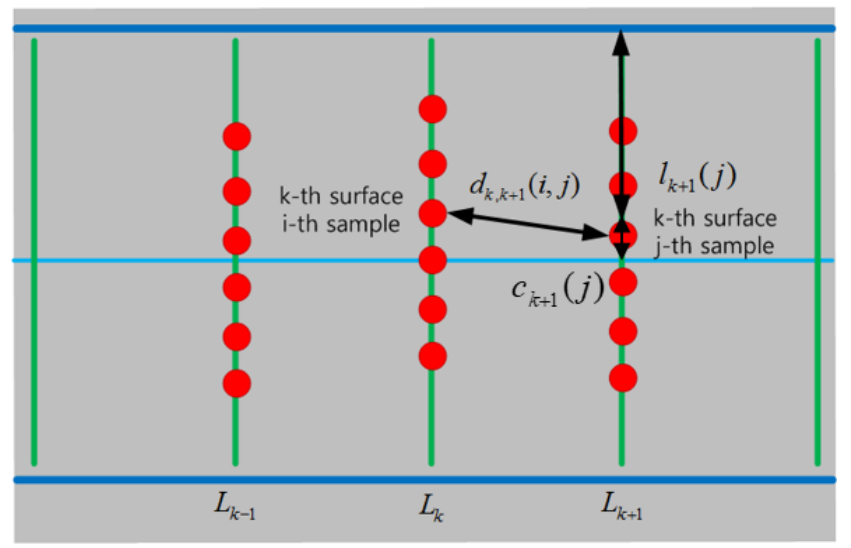

Fig. 9 Example of cost function calculation 
this cost function and selected sample $i$ at $k$-th seeding surface, select sample at $k+1$-th seeding surface as follows

$$
\underset{j}{\operatorname{argmin}}=C_{k, k+1}(i, j)
$$

This method is similar process of dynamic programming because this approach solves a optimization problem by dividing it into several simpler sub-problems, solving each of those sub-problems just once, and storing their solutions. After samples are selected using the above approach to generate a desired local path, this local path is smoothed using B-spline which is composed of a piecewise polynomial function that expressed as a linear combination as follows :

$$
S_{n}(x)=\sum_{i} P_{i} B_{i, n}(t)
$$

where, $P_{i}$ is the seleted samples which should be connected with smooth line to increase path tracking performance. To connect samples smooth enough, cox-deboor recursion formula has been used to derive a piecewise polynomial coefficient $B_{i, n}(t)$ as below ${ }^{(24)}$

$$
\begin{aligned}
& B_{i, 0}(t)= \begin{cases}1 & \text { if } t_{i} \leq t<t_{i+1} \\
0 & \text { otherwise }\end{cases} \\
& B_{i, j}(t)=\frac{t-t_{i}}{t_{i+j}-t_{i}} N_{i, j-1}(t)+\frac{t_{i+j+1}-t}{t_{i+j+1}-t_{i+1}} N_{i+1, j-1}(t)
\end{aligned}
$$

The results of the local path planning is described in Fig. 10. As shown in Fig. 10, As mentioned before, high risk region is marked as yellow grid and safe region is marked as dark blue grid. Also, drivable area which is the boundary of the safe region is marked as black dotted line which is the first grid with maximum potential value. Seeding surfaces and random samples are shown as vertical red solid lines and cross green markers on these lines. Final results of local path planning is red dotted line which connects selected random samples by B-spline.

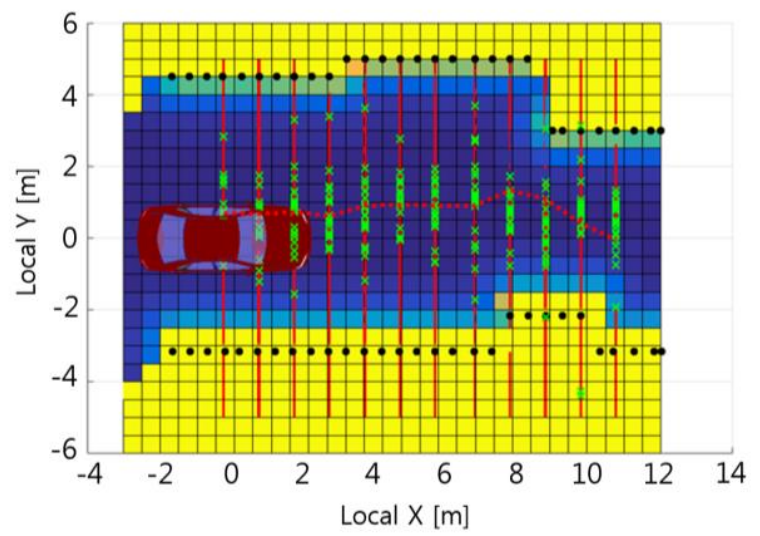

Fig. 10 Sample Geneartion and Local Desired Path

\section{Simulation Results}

The proposed algorithm has been evaluated by MATLAB based computer simulation using the virtual parking lot map and target vehicle. Before planning the desired motion of the target vehicle, appropriate motion of target vehicle and perception model has been constructed to improve reality of the simulation. In this paper, target vehicle's motion is pre-defined not to feedback the motion of the ego vehicle, which makes avoiding collision with target much harder than real driving. Also, perception model gives only relative position and heading angle of target with Gaussian noise which has a standard deviation of $0.2 \mathrm{~m}$ and 5 deg each.

The simulation results is shown in Fig. 11 and 12. To describe the results of the proposed algorithm easily, snapshots of the avoiding an oncoming vehicle scenario are represented in
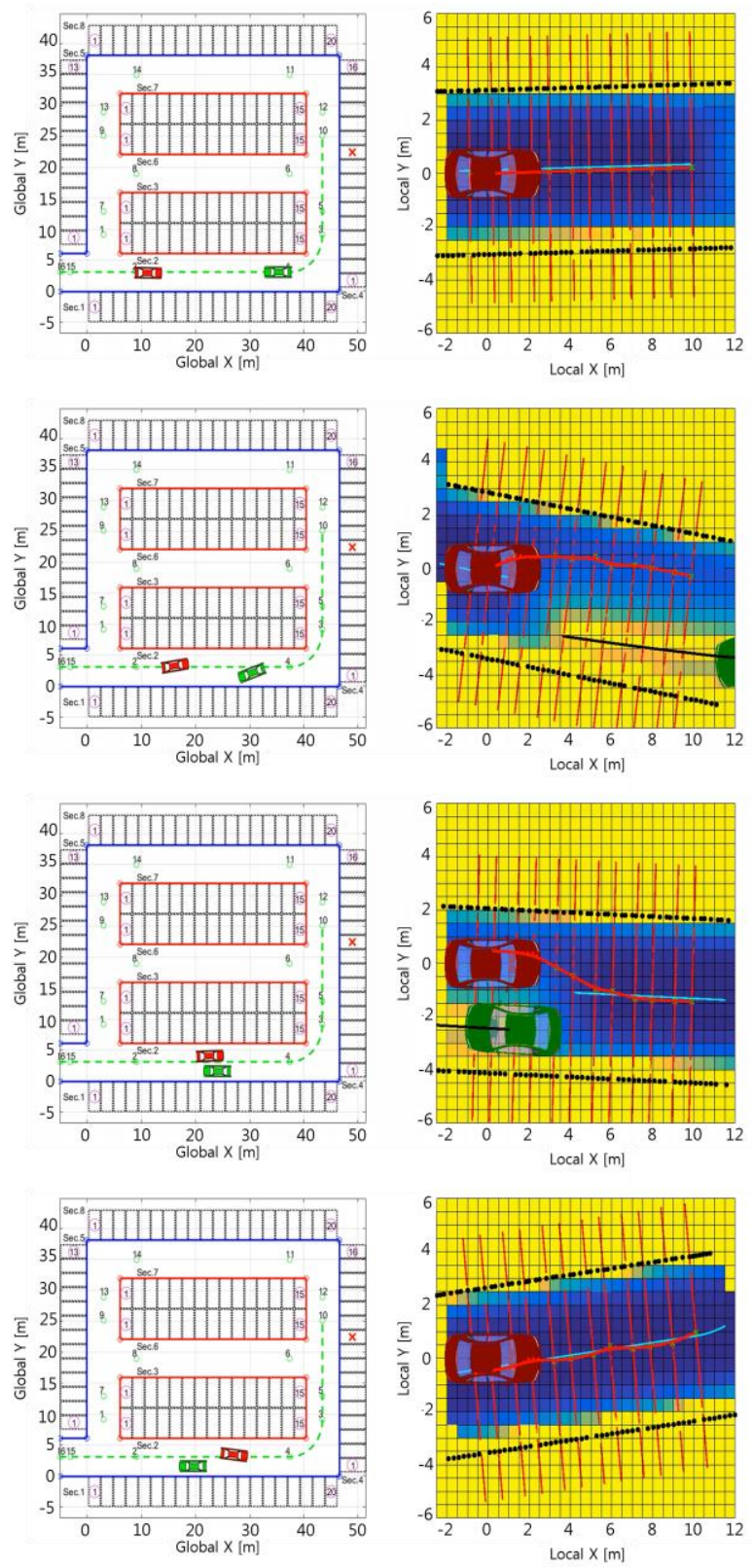

Fig. 11 Snapshot of the Simulation Results 

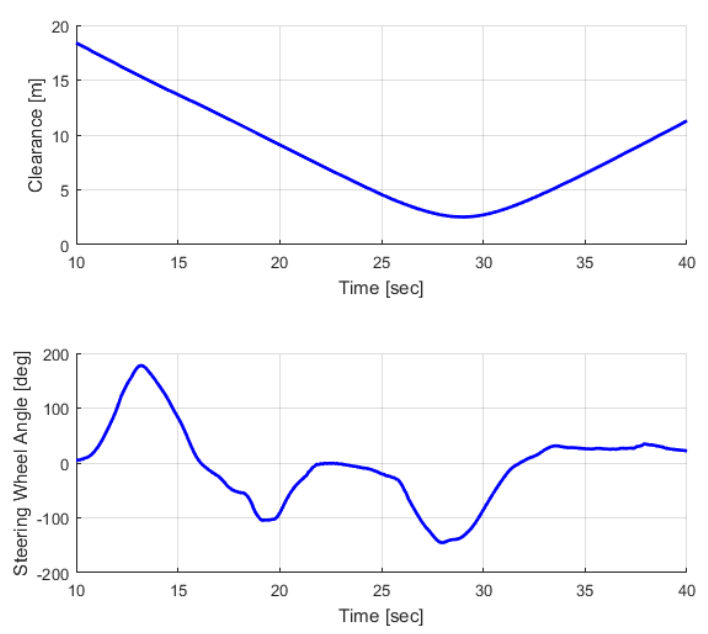

Fig. 12 History of Clearance and Steering Wheel Input

global and local coordinate each. As mentioned before, configuration of the global and local view is maintained same in chapter 3.3. But to show the global path of ego vehicle, green dashed line and cyan solid line is added on global and local plot respectively. One assumption that goal parking lot is given from the infrastructure to generate the global path toward goal parking lot which is marked red cross mark in global plot.

Based on these simulation conditions and assumption, the results of the simulation can be summarized as Fig. 10. In first plot, ego vehicle generate desired local path similar to global path to maximize clearance to boundary of drivable area. This strategy improves the response ability of emergency situations such as a parked vehicle coming out a parking lot suddenly or a car coming from blind spot due to parked vehicle. In this moment, ego vehicle keeps straight because sufficient clearance is secured After several steps, ego vehicle detect the yaw motion of target vehicle and predicted future trajectory of the target vehicle is expressed black solid line in second plot. As can be seen in second plot, high risk region is extended from predicted trajectory of target which makes ego vehicle avoid the oncoming vehicle to the left. Also, heading angle of ego vehicle is smaller than target vehicle because intention inference delay is exist before enough yaw motion of target occurred. After two vehicles crossing each other, ego vehicle return to center of the road without overshoot.

The detail of this simulation is shown in Fig. 11. In this simulation, vehicle speed is maintained as $7 \mathrm{~km} / \mathrm{h}$. The minimum clearance between two vehicle is about $2.5 \mathrm{~m}$ based on the center of the vehicle, which means that clearance between body of two vehicles is maintained above $0.7 \mathrm{~m}$ if we assume the width of the vehicle $1.8 \mathrm{~m}$. Control input, steering wheel angle, is generated as smooth profile while remaining within 200 deg range without oscillation and overshoot. After crossing the target vehicle, parking path design and tracking is described in our previous work ${ }^{(25)}$.

\section{Conclusion}

The motion planning for autonomous valet parking in dynamic environments has been developed to enhance the driver convenience in parking lot. The motion predictor precisely predicts object vehicle's future trajectory and potential filed based drivalble area decision algorithm determine the boundary of the safety region for the local path planning. RRT based path planning algorithm generates safe desired path. Then, LQR path tracking algorithm and model free adaptive controller is used to track the local desired path and desired speed. The performance of the proposed algorithm is evaluated via MATLAB based computer simulation. The results shows that the proposed algorithm guarantees the safety margin between the oncoming vehicle and ego vehicle.

Ongoing work focuses on the extend the inferable intentions to deal with more complex situations in parking lot such as intersection or blind corner. Also, based on these intetions, situation awareness must be added to decide the more sophisticated local path in complex scenarios. After that, vehicle test should be conducted to prove the practicality of the proposed autonomous valet parking algorithm in real parking lot with moving obstacles. To do this, the perception module and localization algorithm should be integrated with the proposed algorithm.

This paper is written based on a proceeding presented at JSAE FAST-zero'17 Meeting

\section{Acknowledgment}

This research was supported by LG Electronics, the Korea Ministry of Land, Infrastructure and Transport, the Korea Agency for Infrastructure Technology Advancement(Project No.: 17TLRP-B117133-02), the brain Korea 21 Plus Project in 2017, and the Institute of Advanced Machines and Design (IAMD), Seoul National University

\section{References}

(1) W. Kim, D. Kim, K. Yi, and H. J. Kim, "Development of a path-tracking control system based on model predictive control using infrastructure sensors," Vehicle System Dynamics, Vol. 50, No. 6, pp. 1001-1023 (2012)

(2) C. Wang, H. Zhang, M. Yang, X. Wang, L. Ye, and C. Guo, "Automatic Parking Based on a Bird's Eye View Vision System," Advances in Mechanical Engineering, Vol. 6, No. 847406 (2015)

(3) M. Chirca, R. Chapuis, and R. Lenain, "Autonomous Valet Parking System Architecture," pp. 2619-2624 (2015)

(4) M. Kyoung-Wook and C. Jeong-Dan, "Design and implementation of an intelligent vehicle system for autonomous valet parking service," pp. 1-6 (2015)

(5) J. Timpner, S. Friedrichs, J. van Balen, and L. Wolf, "kStacks: High-density valet parking for automated vehicles," pp. 895-900 (2015)

(6) R. K. Tendler, "Vehicle backing aid," ed: Google Patents (1990)

(7) B. Z. Shisgal, H. Anderman, Y. Fashchik, and Y. Porat, "Obstacle detection system for vehicles moving in reverse," ed: Google Patents (1996)

(8) K. W. Seo, "Smart parking assist system and control method thereof," ed: Google Patents (2013)

(9) G. S. Aoude, B. D. Luders, J. M. Joseph, N. Roy, and J. P. How, "Probabilistically safe motion planning to avoid dynamic obstacles with uncertain motion patterns," Autonomous Robots, Vol. 35, No. 1, pp. 51-76 (2013)

(10) Y. Kuwata, S. Karaman, J. Teo, E. Frazzoli, J. P. How, and G. Fiore, "Real-Time Motion Planning With Applications to Autonomous Urban Driving," IEEE Transactions on Control Systems Technology, Vol. 17, No. 5, pp. 1105-1118 (2009) 
(11) J. Lee, B. Kim, J. Seo, K. Yi, J. Yoon, and B. Ko, "Automated Driving Control in Safe Driving Envelope Based on Probabilistic Prediction of Surrounding Vehicle Behaviors," SAE International Journal of Passenger CarsElectronic and Electrical Systems, Vol. 8, No. 2015-01-0314, pp. 207-218 (2015)

(12) J. Schulman et al., "Motion planning with sequential convex optimization and convex collision checking," The International Journal of Robotics Research, Vol. 33, No. 9, pp. 1251-1270 (2014)

(13) A. Gray, Y. Gao, T. Lin, J. K. Hedrick, H. E. Tseng, and F. Borrelli, "Predictive control for agile semi-autonomous ground vehicles using motion primitives," in 2012 American Control Conference (ACC), pp. 4239-4244: IEEE (2012)

(14) J. Seo and K. Yi, "Robust Mode Predictive Control for Lane Change of Automated Driving Vehicles," SAE Technical Paper, No.2015-0148-7191 (2015)

(15) A. Gray, Y. Gao, T. Lin, J. K. Hedrick, and F. Borrelli, "Stochastic predictive control for semi-autonomous vehicles with an uncertain driver model," in 16th International IEEE Conference on Intelligent Transportation Systems (ITSC 2013), pp. 2329-2334: IEEE (2013)

(16) S. S. Ge and Y. J. Cui, "Dynamic motion planning for mobile robots using potential field method," Autonomous Robots, Vol. 13, No. 3, pp. 207-222 (2002)

(17) R. Matsumi, P. Raksincharoensak, and M. Nagai, "Autonomous braking control system for pedestrian collision avoidance by using potential field," IFAC Proceedings Volumes, Vol. 46, No. 21, pp. 328-334 (2013)

(18) S. Shimoda, Y. Kuroda, and K. Iagnemma, "Potential field navigation of high speed unmanned ground vehicles on uneven terrain," in Proceedings of the 2005 IEEE International Conference on Robotics and Automation, pp. 2828-2833: IEEE (2005)

(19) K. Kim, B. Kim, K. Lee, H. Chong, B. Ko, and K. Yi, "Vehicle motion control for integrated risk management of automated vehicle," Proceedings of FAST-zero' 15: 3rd International Symposium on Future Active Safety Technology Toward zero traffic accidents, pp.229-234 (2015)

(20) S. Yoon, S.-E. Yoon, U. Lee, and D. H. Shim, "Recursive Path Planning Using Reduced States for Car-Like Vehicles on Grid Maps," IEEE Transactions on Intelligent Transportation Systems, Vol. 16, No. 5, pp. 2797-2813 (2015)

(21) B. Li, and Z. Shao, "A unified motion planning method for parking an autonomous vehicle in the presence of irregularly placed obstacles," Knowledge-Based Systems Vol.86, pp. 1120 (2015)

(22) H. Kim, and K. Yi, "Combined throttle and brake control for vehicle cruise control: a model free approach," Proceedings of 2013 IEEE Intelligent Vehicles Symposium (IV), pp. 859-864 (2013)

(23) E. Mazor, A. Averbuch, Y. Bar-Shalom, and J. Dayan, "Interacting multiple model methods in target tracking: a survey." IEEE Transactions on aerospace and electronic systems Vol. 34, No. 1, pp. 103-123 (1998)

(24) E. TY. Lee, "A simplified B-spline computation routine." Computing Vol. 29, No. 4, pp. 365-371 (1982)

(25) Y. Jeong, S. Kim, K. Yi, S. Lee, and B. Jo, "Design and Implementation of Parking Control Algorithm for Autonomous Valet Parking," SAE Technical Paper, No. 201601-0146 (2016) 